\title{
Mechanics of Nanowires
}

Rodney S. Ruoff*, Xinqi Chen, Dmitriy Dikin, Weiqiang Ding, Ming-Feng Yu $\mathrm{u}^{\mathrm{a}}$, Gregory J. Wagner

Department of Mechanical Engineering, Northwestern University, 2145 Sheridan Road, Evanston, IL 60208

ayvex Corporation, Advanced Technologies Group, 1321 North Plano Road, Richardson, TX 75081

Carbon nanotubes (NTs) and boron and $\mathrm{SiO}_{2}$ nanowires (NWs) have many potential applications in scientific and industrial fields due to their size and expected excellent mechanical and electrical properties. Here we present the further development of the mechanical resonance method for characterizing the mechanical properties of such nanowires.

A homemade nanomanipulation testing stage (shown in Fig. 1), having $X, Y, Z$ and rotational degrees of freedom, was used in these measurements of the resonance response of C NTs and SiO2 NWs. The measurements were made in an SEM chamber (Hitachi S4500 FEG-SEM). The NTs or NWs were attached on a Pt/Ir wire with conductive carbon tape. A tungsten tip was brought into proximity and an ac electric field was applied between the Pt/Ir wire and the tungsten tip. The NT or NW could be driven into resonance by varying the frequency of the electric field. The bending modulus of multiwall $\mathrm{C}$ NTs and $\mathrm{SiO}_{2}$ NWs were obtained by fitting the measured resonate frequencies. $[1,2]$ In addition to this method of electromechanical resonance excitation, the resonance could be mechanically excited by a piezoelectric oscillator (without the presence of an applied electric field). Figure 2 shows a $\mathrm{SiO}_{2} \mathrm{NW}$ on resonance.

Parametric resonance, a resonance phenomenon resulting from an oscillation system with a time-varying coefficient, has found wide applications in nonlinear optics and electronics for detecting and amplifying small signals. We also realize such resonance with boron nanowires. Resonance at drive frequencies near $2 f_{0} / n$, where $f_{0}$ is the fundamental resonance of the nanowire, for $n$ from 1 to 4 were observed inside a scanning electron microscope and analyzed. These results, of fundamental interest because they are likely representative of a ubiquitous response of nanoscale systems that intrinsically have smaller damping than macroscale systems, are also of practical interest for applications in the sensor field. Thus we will devote some time developing the theory, and method of modeling, for the audience. ONR MIS and NASA support for current work, NSF support for building tool.

\section{References:}

[1] P. Poncharal, Z. L. Wang, D. Ugarte, W. A. de Heer, Science, 283 (1999) 1513.

[2] Z. L. Wang, R. P. Gao, P. Poncharal, W. A. de Heer, Z. R. Dai, Z. W. Pan, Materials Science and Engineering C, 16 (2001) 3. 


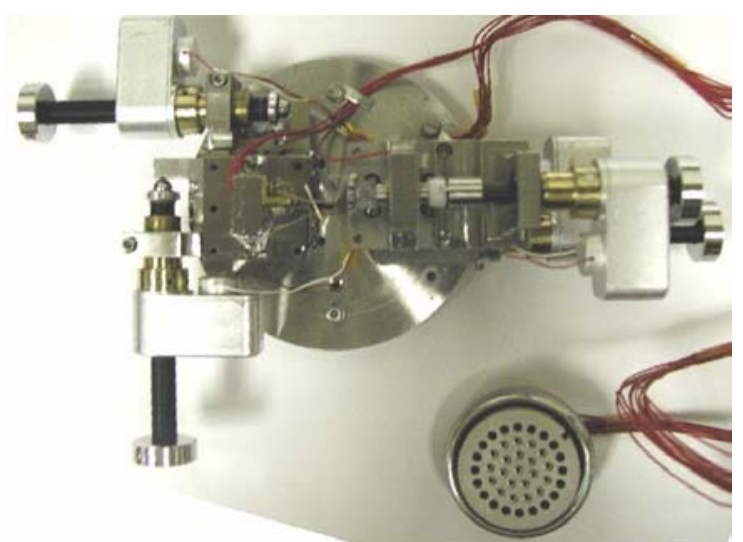

Figure 1. Homemade nanomanipulation testing stage.

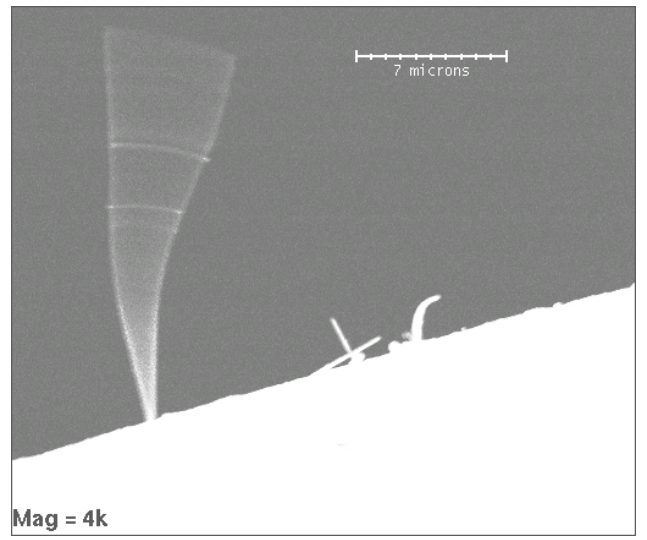

Figure 2. Resonate vibration of a $\mathrm{SiO} 2$ nanowire excited by an ac electric field; a similar resonance response, but not identical, is achieved by mechanically driving via direct physical contact with an oscillating piezoelectric. 